\title{
Diferenças biomecânicas entre a técnica lingual e a labial*
}

\author{
André da Costa Monini**, Luiz Gonzaga Gandini Jr***,

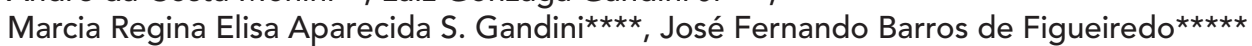

\begin{abstract}
Resumo
Pela sua característica essencialmente estética, o aparelho lingual vem despertando interesse por parte dos ortodontistas e da comunidade em geral. O crescente mercado dos serviços de beleza, em todos os setores (médicos, odontológicos, cosméticos), influencia o ressurgimento da técnica lingual. Biomecanicamente diferente da técnica convencional, a técnica lingual necessita de um conhecimento e treinamento diferenciado do profissional que a executa. Verificar as diferenças, encontradas na literatura, com relação às duas técnicas é o objetivo deste trabalho, como parte do processo de amadurecimento e treinamento profissional confirmando a técnica lingual como mais uma opção segura de tratamento ortodôntico.
\end{abstract}

Palavras-chave: Técnica lingual. Braquetes invisíveis. Braquetes estéticos.

\section{INTRODUÇÃO}

O aparelho lingual remonta de meados da década de 70 quando Fujita, no Japão, trabathou no desenvolvimento de um braquete lingual $^{1}$. Na mesma época, Kurz, nos Estados Unidos, criou seu próprio aparelho lingual fazendo adaptações num aparelho convencional ${ }^{1}$. Desde então, um aparelho próprio para a superfície lingual foi desenvolvido e modificado, por varias companhias, diversas vezes até se chegar nos modelos atuais.

$\mathrm{Na}$ década de 80 muitos pacientes em todo o mundo procuraram tratamento desta forma devido a óbvia vantagem do aparelho não ser prontamente visto. Entusiasmados com esta altíssima demanda de pacientes muitos ortodon- tistas se lançaram a tratar usando esta filosofia e os problemas começaram a surgir, levando a um descrédito da técnica.

Atualmente observa-se um novo interesse pela mesma, desta vez com mais cautela por parte dos profissionais.

A falta de treinamento e de conhecimento científico sobre o comportamento do aparelho lingual prejudicou o desenvolvimento da técnica fazendo com que, ainda hoje, pouca informação consistente seja encontrada na literatura. Para não incorrer nos mesmos erros do passado, o novo interesse pela técnica deve vir acompanhado de prudência e formação técnico-científica por parte do ortodontista. Como parte deste processo, o presente trabalho objetiva ve-

* Resumo da Monografia apresentada à Faculdade de Odontologia de Araraquara - UNESP como parte dos requisitos para obtenção do titulo de Especialista em Ortodontia.

** Especialista em Ortodontia e estagiário da Disciplina de Ortodontia - UNESP - Araraquara

*** Professor Assistente-doutor e Chefe do Departamento de Clinica Infantil - UNESP - Araraquara. Adjunct Clinical Assistent Professor - Baylor College of Dentistry - Dallas - USA.

**** Professora Voluntária do Departamento de Clínica Infantil - UNESP - Araraquara. Adjunct Clinical Assistent Professor - Saint Louis University - USA

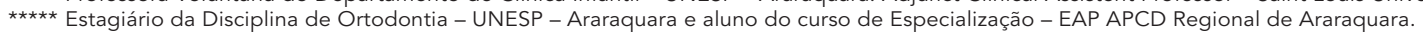


rificar, revisando a literatura, as características biomecânicas que diferenciam a técnica lingual da convencional.

\section{REVISÃO DE LITERATURA E DISCUSSÃO}

O efeito imediato produzido pelo aparelho lingual é a abertura da mordida resultado da oclusão dos incisivos inferiores no plano de mordida dos braquetes dos incisivos superiores $1,2,7,10,12,13,15,17,18,21,24$ (Fig. 1, 2). Esta abertura provoca rotação para baixo e para trás da mandíbula induzindo uma tendência à classe $\mathrm{II}^{10,12,24,28}$ (Fig. 3-6). Mesmo uma classe I, com o alinhamento e nivelamento o molar pode mudar sua relação para classe II principalmente em paciente dólico faciais ${ }^{28}$.

Assim, a utilização de aparelho extrabucal pode se tornar crítica, pela característica essen-

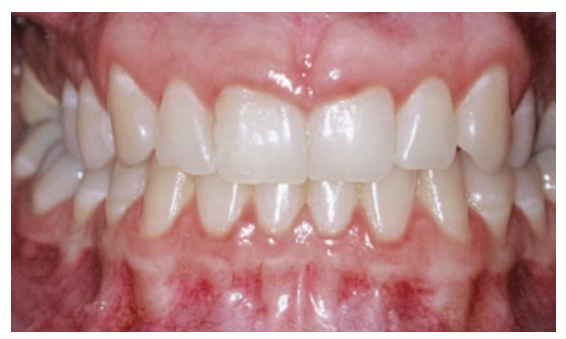

FIGURA 1 - Vista frontal prévia à colagem dos braquetes.

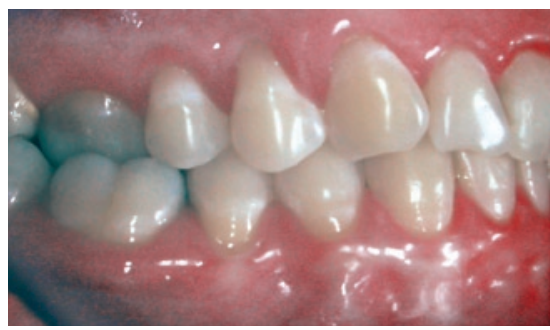

FIGURA 3 - Vista lateral esquerda prévia à colagem dos braquetes.

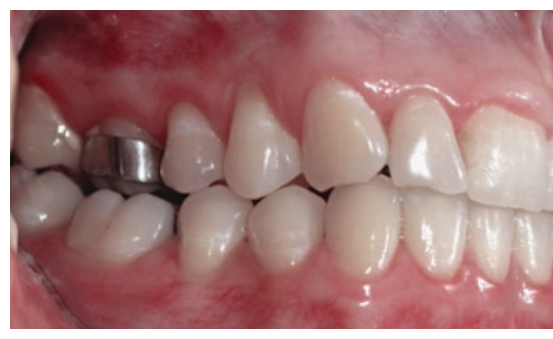

FIGURA 5 - Vista lateral esquerda após a colagem dos braquetes. cialmente estética do aparelho, e a decisão de extração dos primeiros pré-molares superiores pode se tornar mais freqüente que casos tratados com a técnica convencional ${ }^{12,24}$. A desoclusão posterior remove dois fatores primários de ancoragem, a oclusão dentária e a força da musculatura ${ }^{12}$ facilitando, porém a correção de mordida profunda, da mordida cruzada e verticalização de molares².

Existe uma preocupação se a desoclusão posterior provocaria uma extrusão dos dentes posteriores levando a um aumento da dimensão vertical. No entanto, Kelly ${ }^{15}$ não observou aumento do ângulo do plano mandibular (FMA), em seus casos de consultório, ao contrário houve diminuição em $2^{\circ}$, em média devido à combinação de intrusão dos incisivos superiores e inferiores. Smith ${ }^{26}$ reporta dois casos tratados

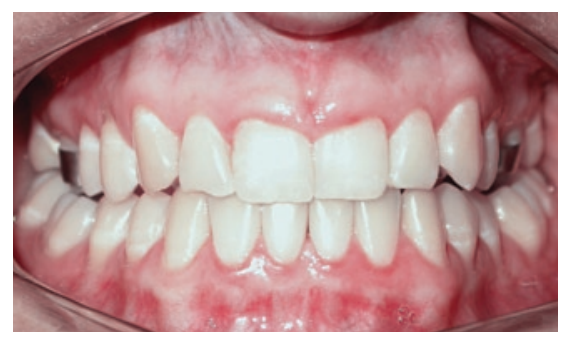

FIGURA 2 - Vista frontal após a colagem dos braquetes.

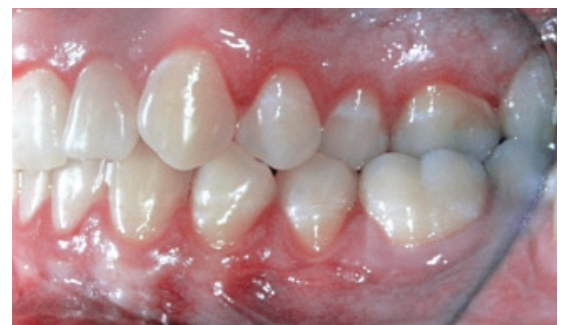

FIGURA 4 - Vista lateral direita prévia à colagem dos braquetes.

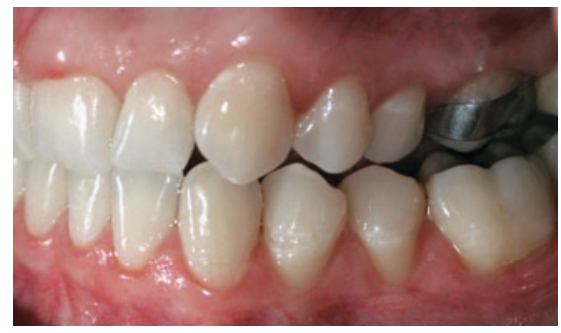

FIGURA 6 - Vista lateral direita após a colagem dos braquetes. 
com extrações e observou diminuição do ângulo do plano mandibular em um caso e não houve alteração no outro. Alexander et al. ${ }^{1}$ também não observaram mudanças no ângulo do plano mandibular. Kurz ${ }^{19}$ relata um caso com quatro extrações sem alterações no plano mandibular.

Kurz e Gorman ${ }^{17}$ reportaram dois casos sem extrações sendo que em um caso o FMA aumentou $1^{\circ}$ e no outro permaneceu constante. Takemoto e Scuzzo ${ }^{28}$ recomendam colocar resina nas cúspides vestibulares dos molares inferiores para se estabelecer a oclusão e assim evitar a extrusão dos dentes posteriores. Fujita ${ }^{9}$ sugere montar o arco inferior primeiro e intruir os incisivos até poder colar o arco superior sem interferências. Em quatro pacientes tratados desta forma demonstrou diminuição do ângulo FMA em $3^{\circ}$ em dois deles, aumento em $1^{\circ} \mathrm{em}$ um caso e outro se manteve constante. Trabalhando com a recomendação de Fujita ${ }^{9}$, Hong, Hong e $\mathrm{Koh}^{14}$ verificaram aumento de $0.5^{\circ} \mathrm{em}$ cinco dos oito pacientes tratados. Nos outros três o FMA permaneceu o mesmo em um caso e diminuiu, em média, $0.9^{\circ}$ nos outros dois.

Em nove pacientes tratados sem extração, Fulmer e Kuftinec ${ }^{10}$ encontraram que o ângulo FMA aumentou $1.5^{\circ}$, em média, e pela medida SNGoGn aumentou $1.1^{\circ}$. Em treze pacientes tratados com quatro extrações, o FMA aumentou $0.6^{\circ}$, SNGoGn aumentou $0.3^{\circ}$ e o ângulo da altura facial ântero-inferior aumentou $0.9^{\circ}$. Considerando-se os dois grupos, com e sem extrações, o FMA diminuiu $2^{\circ}$ ou mais em quatro casos e o SNGoGn diminuiu $2^{\circ}$ ou mais em somente um caso. $\mathrm{O}$ ângulo FMA aumentou $2^{\circ}$ ou mais em onze casos, e SNGoGn aumentou $2^{\circ}$ ou mais em oito casos. Conseqüentemente examinando cada caso individualmente mais aumentos que diminuições no plano mandibular ocorreram, mas muitos pacientes exibiram pequena mudança.

Gorman e Smith ${ }^{13}$ fizeram um trabalho comparando os efeitos do tratamento pela téc- nica lingual e pela convencional. Encontraram aumento da altura facial ântero-inferior (AFAI) de $0.8 \mathrm{~mm}$ e $0.4^{\circ}$ de abertura do eixo Y nos casos tratados com extração e usando aparelho convencional, enquanto nos casos tratados com lingual, também com extrações, a AFAI aumentou $0.9 \mathrm{~mm}$ e $0.2^{\circ}$ no eixo Y. Nos casos sem extrações, o tratamento com aparelho convencional mostrou aumento na AFAI de $1.1 \mathrm{~mm}$ e abertura do eixo Y de $0.3^{\circ}$. Nos casos com aparelho lingual o aumento da AFAI foi de $0.7 \mathrm{~mm}$ e abertura do eixo $\mathrm{Y}$ de $0.5^{\circ}$. As medidas cefalométricas deste estudo revelaram não ter diferenças estatisticamente significantes no tratamento com o lingual e o convencional.

Avaliando o padrão de resposta dos dentes às forças ortodônticas aplicadas no braquete lingual comparando ao convencional percebese que o padrão de movimento é diferente. Forças intrusivas num braquete lingual tendem a intruir os incisivos com menos inclinação comparando com o mesmo tipo de força por vestibular $^{29}$ (Fig. 7). O resultado conseguido pelo aparelho lingual pode ser explicado pela maior proximidade do braquete lingual com o centro de resistência do dente ${ }^{11,28}$. Deste modo, momentos criados por forças aplicadas no braquete lingual comparados com braquete convencional são sempre menores, gerando menos efeito de inclinação ${ }^{11,28}$.

Geron, Romano e Brosh ${ }^{11}$ avaliaram o efeito de forças intrusivas e extrusivas no movimento de um incisivo superior, em braquetes por lingual e vestibular, através de um modelo matemático. A inclinação do incisivo foi relacionada a uma linha perpendicular ao plano oclusal variando de $35^{\circ}$ de retro inclinação a $45^{\circ}$ de proclinação, sendo que a $0^{\circ}$ o dente estava perpendicular ao plano oclusal. Os resultados mostraram que momento jogando a raiz para lingual foi conseguido com força intrusiva num aparelho convencional com o dente inclinado de $20^{\circ}$ de retro inclinação até $45^{\circ}$ de proclina- 


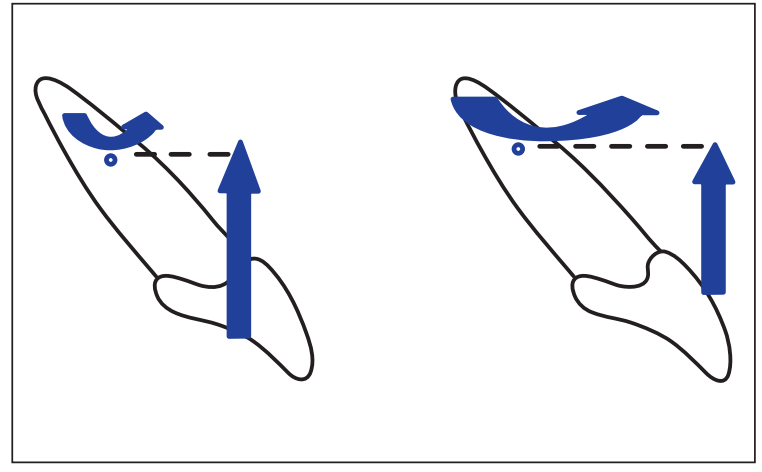

FIGURA 7 - Magnitude dos momentos gerados pela aplicação da força por lingual e vestibular.

ção. Usando aparelho lingual o momento jogando a raiz para lingual só foi conseguido quando o dente estava, pelo menos, proclinado mais que $20^{\circ}$.

A maior proximidade do braquete lingual com o centro de resistência do dente também provoca uma transmissão de força mais intensa ao ligamento periodontal em relação à técnica convencional $^{6,27}$ e em associação com a menor distância interbraquete ${ }^{1,6,7,10,13,20,21,24,25}$ acabam por, praticamente, obrigar o ortodontista a utilizar fios mais finos e resilientes ${ }^{1,2,15,20,21,25}$. Do ponto de vista clínico a chance de maior reabsorção radicular e maior área de hialinização ocorre com a técnica lingual, então o ortodontista deve ter cuidado de, em condições similares, fazer menor ativação no aparelho lingual que no convencional ${ }^{6}$. As figuras 8,9 e 10 mostram uma comparação de equivalência de força entre os arcos vestibular e lingual.

Chaconas, Caputo e Brunetto ${ }^{6}$ avaliaram a transmissão das forças pelo aparelho lingual e pelo convencional num modelo fotoelástico e a comparação foi feita com fio de nivelamento 0,016" de beta titânio e arco de retração 0,016" x 0,016" "Blue Elgiloy". Concluíram que a distribuição do stress foi similar no aparelho lingual e no convencional, mas a intensidade da força produzida pelo aparelho lingual foi maior (Fig. 11-16).
Um resultado semelhante foi conseguido por Sung, Baik, Moon, Yu e Cho ${ }^{27}$ trabalhando com método de elemento finito em modelo tridimensional avaliando a retração de canino com os dois tipos de aparelhos. As diferenças na intensidade da força tendem a diminuir na região posterior ao primeiro pré-molar.

O simples fato de se colar braquete por lingual já traz imediatas diferenças com relação ao aparelho convencional como a menor distancia interbraquete ${ }^{1,6,7,10,13,20,21,24,25}$, a forma do $\operatorname{arco}^{1,9,14,21,22}$ e a diferença anatômica da superfície lingual $8,10,11,17,18,21,22,23,24,28$.

Em média, a distância interbraquete do aparelho convencional quando comparada com o lingual é de 1.47:120. Como a rigidez do fio é inversamente proporcional ao cubo do comprimento, a diminuição da distância interbraquete aumenta significantemente as forças aplicadas no dente provocando uma diferença de mais de 3 vezes para fios iguais colocados em arcos por lingual e por vestibular ${ }^{4,5,20}$. Isto contribui para a transmissão mais intensa da força ao dente pela técnica lingual.

A necessidade de se utilizar fios mais resilientes dificulta o tratamento ortodôntico por lingual que envolva a utilização de elásticos intermaxilares ou extrações. Da mesma forma que o tratamento convencional, para o fechamento de espaços e para a utilização de elásticos intermaxilares são necessários arcos consolidados e com fios retangulares $2,12,24,25,28$. Deste modo, problemas de vestibularização de pré-molares em áreas de extração ${ }^{12,24,25,28}$ e inclinação dos dentes anteriores com perda do controle do torque $^{12,16,25,26,27,28}$ são mais freqüentes na técnica lingual.

Incorporação do segundo molar no $\operatorname{arco}^{24}$, uso de barra palatina ${ }^{2,12,24,28}$, colagem de braquetes por vestibular nos dentes posteriores (técnica mista $)^{2,12,25}$ e colocar uma curvatura para lingual no arco de fechamento ${ }^{12,24,25,27,28}$ são soluções apresentadas e praticamente obrigatórias 


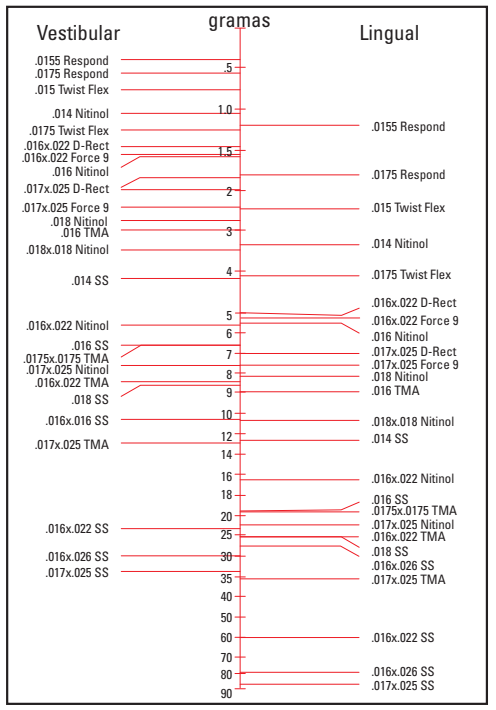

FIGURA 8 - Equivalência de força e rigidez dos arcos linguais (direita) com os convencionais (esquerda) para discrepâncias dentais em primeira ordem. Fonte: Modificado de Moran ${ }^{20}$.

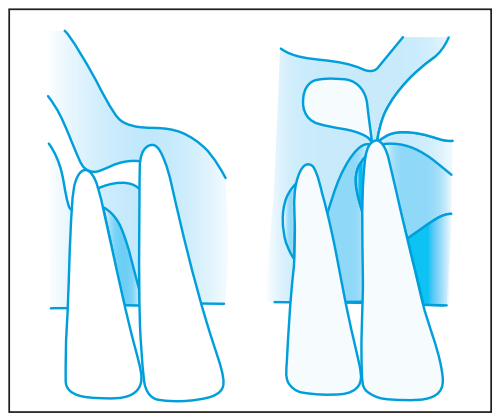

FIGURA 11 - Transmissão de força pelos arcos de alinhamento nos incisivos superiores: à esquerda aparelho convencional e à direita aparelho lingual. Fonte: CHACONAS $^{6}$.

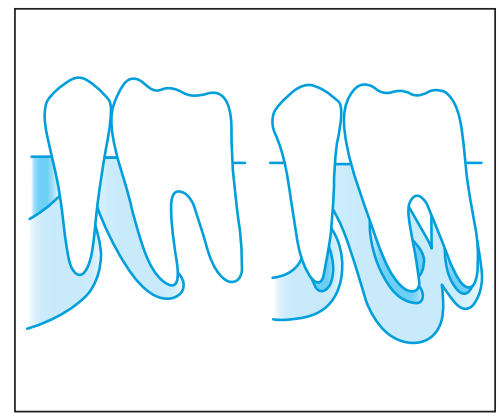

FIGURA 14 - Transmissão de força pelos arcos de alinhamento nos posteriores inferiores: à esquerda aparelho convencional e à direita aparelho lingual. Fonte: CHACONAS $^{6}$.

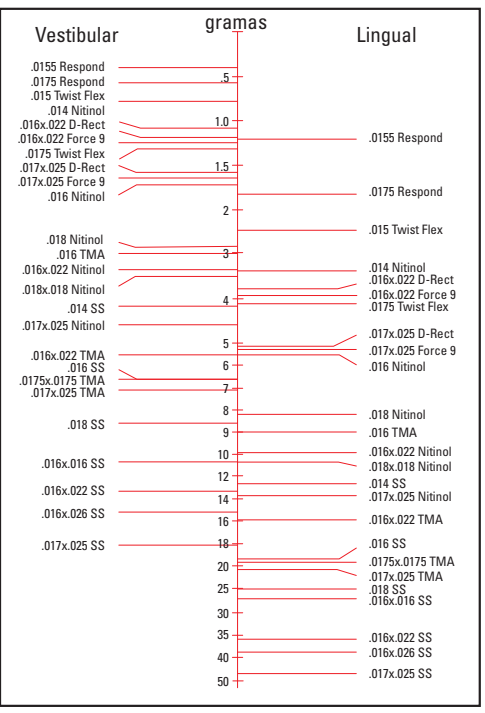

FIGURA 9 - Equivalência de força e rigidez dos arcos linguais (direita) com os convencionais (esquerda) para discrepâncias dentais em segunda ordem. Fonte: Modificado de Moran ${ }^{20}$.

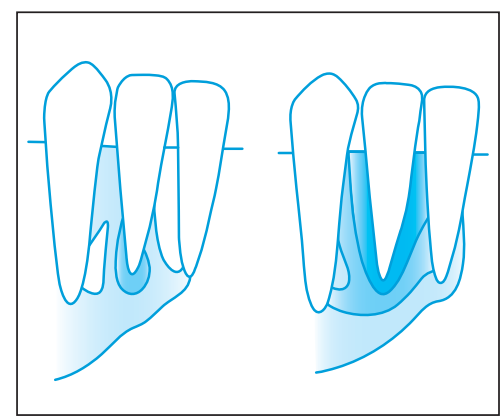

FIGURA 12 - Transmissão de força pelos arcos de alinhamento nos incisivos inferiores: à esquerda aparelho convencional e à direita aparelho lingual. Fonte: CHACONAS ${ }^{6}$.

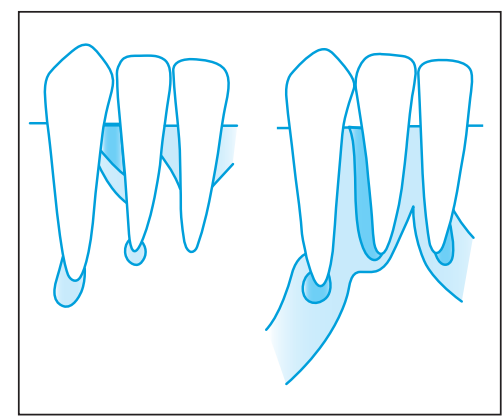

FIGURA 15 - Transmissão de força pelos arcos de retração nos incisivos inferiores: à esquerda aparelho convencional e à direita aparelho lingual. Fonte: CHACONAS $^{6}$

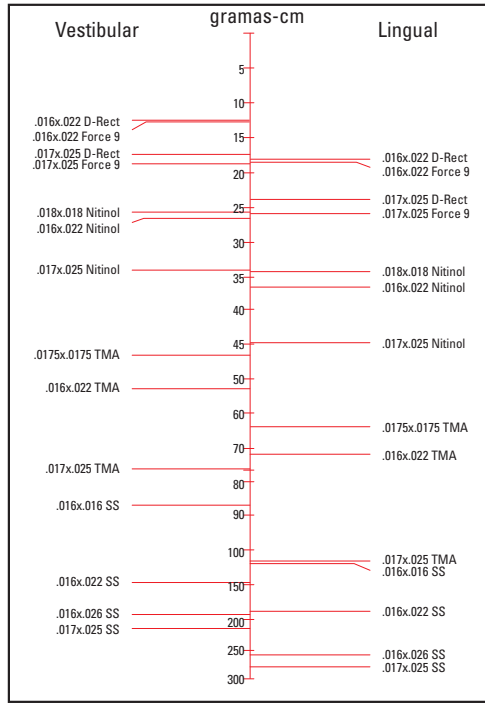

FIGURA 10 - Equivalência de força e rigidez dos arcos linguais (direita) com os convencionais (esquerda) para discrepâncias dentais em terceira ordem. Fonte: Modificado de Moran ${ }^{20}$.

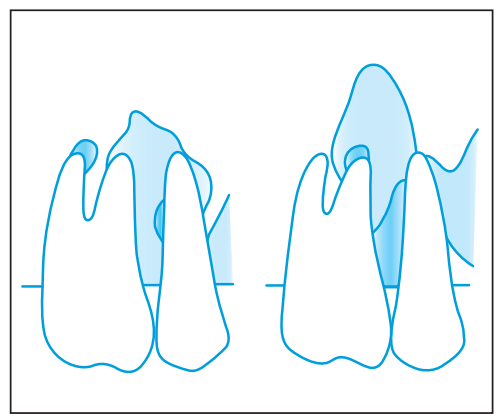

FIGURA 13 - Transmissão de força pelos arcos de alinhamento nos dentes posteriores superiores: à esquerda aparelho convencional e à direita aparelho lingual.

Fonte: $\mathrm{CHACONAS}^{6}$.

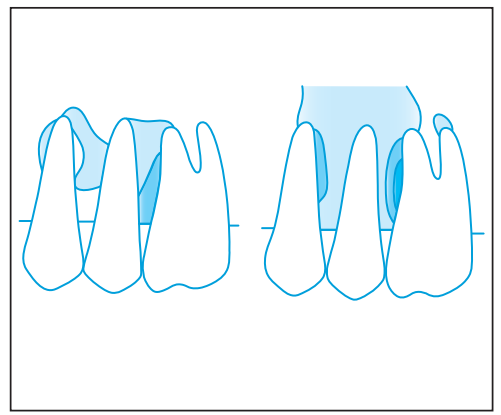

FIGURA 16 - Transmissão de força pelos arcos de retração nos posteriores superiores: à esquerda aparelho convencional e à direita aparelho lingual. Fonte: CHACONAS ${ }^{6}$. 
para tratamento com extrações na técnica lingual. Como as forças de retração estão por lingual do centro de resistência, os dentes tendem a mover no sentido inverso do que ocorre por vestibular, ou seja, os molares tendem a girar com a mesial para vestibular ${ }^{27}$ (Fig. 17).

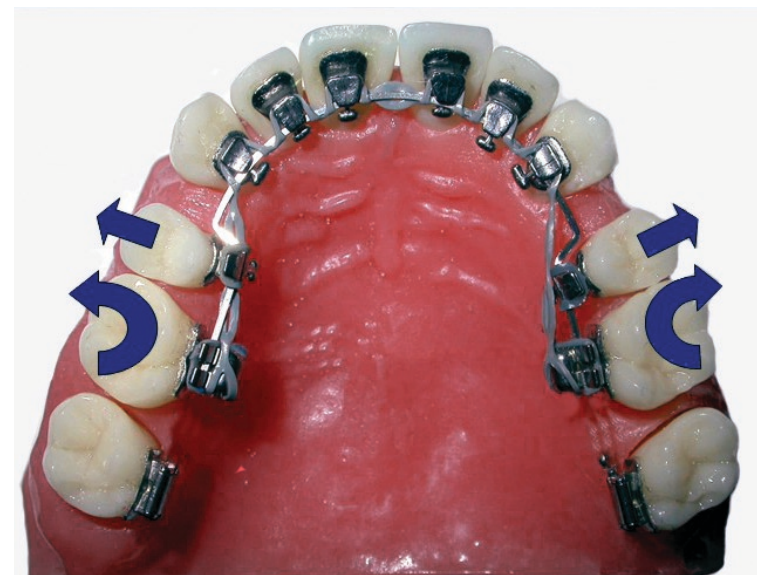

FIGURA 17 - Efeito colateral da aplicação de forças de retração por lingual.
Retração em massa dos seis dentes anteriores normalmente é a escolhida para os tratamentos com aparelho lingual pois a retração parcial de caninos produziria um espaço antiestético distal aos laterais, normalmente não desejado pelo paciente, com aparelho lingual 12,12,25,27,28. Em termos de mecânica, a completa retração dos caninos diminui a distância interbraquete entre o canino e o pré-molar devido ao "inset" no arco distal ao canino, e desta forma, diminui-se a quantidade possível de ativação na mecânica de deslizamento ou por alças ${ }^{28}$.

Outra característica oposta à técnica convencional é a tendência do arco sair do "slot" durante as ativações já que por vestibular o efeito é o inverso $2,19,25$. Deste modo, é necessário que o arco esteja firmemente amarrado pelo método do "double over tie"2,25,28 (Fig. 18-25).

De um modo geral o arco apresenta uma dobra próximo de $90^{\circ}$ entre o canino e o pri-

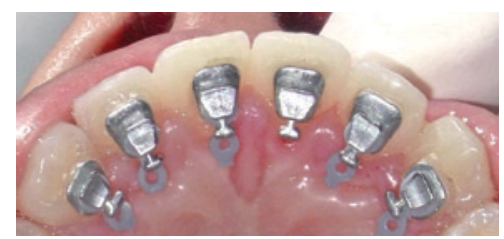

FIGURA 18 - Braquete sem ligadura elástica.

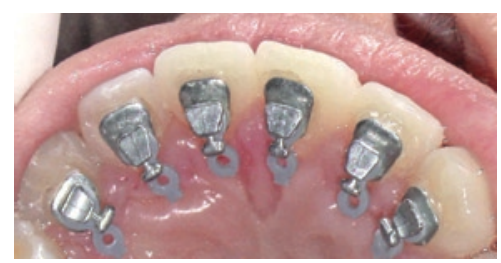

FIGURA 21 - Segundo elo para cervical.

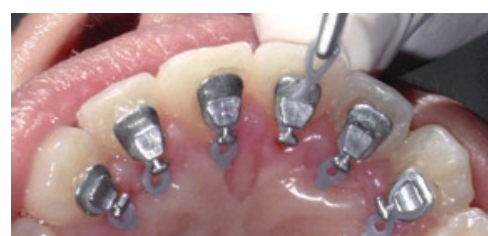

FIGURA 19 - Inserção de um elo da ligadura elástica.

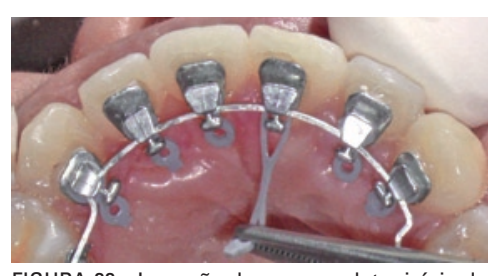

FIGURA 22 - Inserção do arco no slot e início do double over tie.

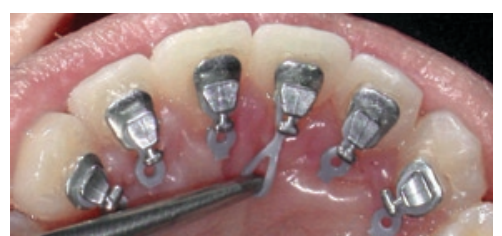

FIGURA 20 - Manter o segundo elo para cervical.

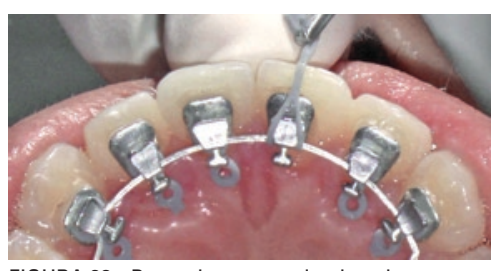

FIGURA 23 - Puxando o segundo elo sobre 0 arco.

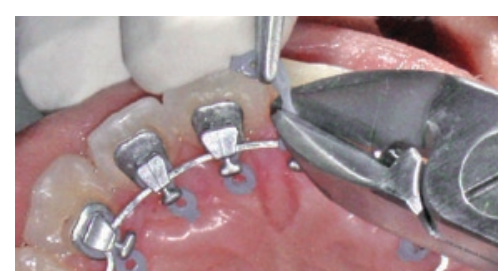

FIGURA 24 - Cortando o segundo elo.

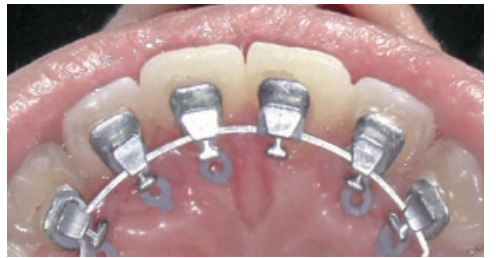

FIGURA 25 - Final do double over tie. 


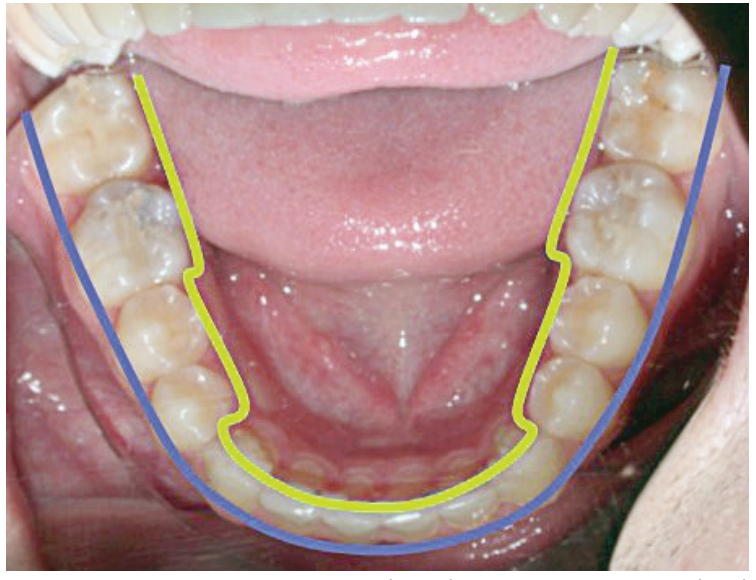

FIGURA 26 - Aparência do arco lingual (verde) e do arco convencional (azul) na arcada inferior.

meiro pré-molar com suficiente tamanho para compensar a diferença de espessura vestíbulo lingual entre eles podendo apresentar, também, um degrau menor entre o segundo pré-molar e o primeiro molar ${ }^{1,2,7,25}$. A aparência final do arco lembra um cogumelo ${ }^{1,9,14,21,22}$ (Fig. 26, 27).

$\mathrm{O}$ aparelho lingual pode ser expansivo por natureza devido à insuficiente constrição do arco nos dentes anteriores ${ }^{1,2,12,24,15}$. Se o arco for ligeiramente maior que a curvatura anterior ele pode produzir uma força extra de expansão ${ }^{2}$.

A dobra de primeira ordem entre o canino e o primeiro pré-molar pode atuar como "stop",24 impedindo o deslizamento do arco à medida que o alinhamento vai ocorrendo e também pode providenciar uma força expansiva pela incorporação de fio no arco deixando-o mais flexível ${ }^{27}$.

Segundo Smith, Gorman, Kurz e Dunn ${ }^{25}$, os casos clínicos que relatam expansão com a técnica lingual provavelmente sejam devido a iatrogenia, ou seja, insuficiente constrição do arco durante o alinhamento e nivelamento ou colocação da dobra entre o canino e o pré-molar muito afastado da distal atuando como "stop" do deslizamento do arco.

A colagem direta dos braquetes na superfície lingual é praticamente inviável pois existem diferenças de espessuras entre os dentes e a super-

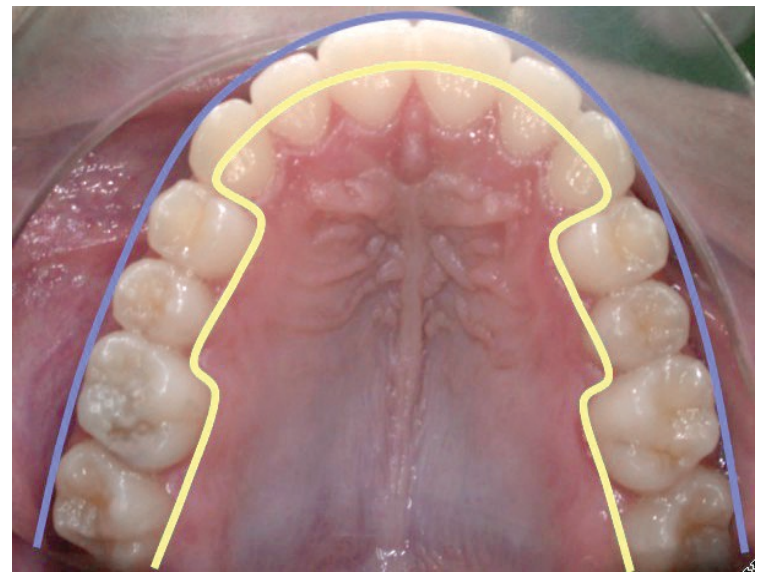

FIGURA 27 - Aparência do arco lingual (amarelo) e do arco convencional (azul) na arcada superior.

fície lingual é muito mais propensa a apresentar alterações de forma, tamanho e irregularidades superficiais, afetando sensivelmente, o correto posicionamento dos braquetes $8,11,22,23,24$. Além disso, pequenas alterações neste posicionamento podem provocar grandes efeitos indesejáveis. Braquetes colados na mesma altura por vestibular e lingual podem variar o posicionamento do dente nos três planos do espaço $0^{8,10,17,18,21,23,28}$ (Fig. 28, 29). Alem disso, pequenas variações cérvico-oclusais na face lingual alteram consideravelmente o torque dos braquetes.

Assim, conceito "straight-wire" é falho para o tratamento com aparelho lingual. Uma das soluções é a colocação de compensações de resina na base dos braquetes associada à colagem indireta ${ }^{1,7,8,21,23,24,28}$ (Fig. 30, 31).

O aparelho lingual pode ser um armamento valioso e contemporâneo para o ortodontista que trata de um segmento da população que é exigente ${ }^{1,9,10,20}$. O ortodontista precisa estar familiarizado com as características do aparelho para diminuir e eliminar problemas, selecionar casos específicos e instruir os pacientes sobre as limitações ${ }^{2,24}$. Entender e aplicar princípios biomecânicos no tratamento aumenta a eficácia do aparelho e simplifica o tratamento, pois obtém movimentos dentários mais previsíveis e com 


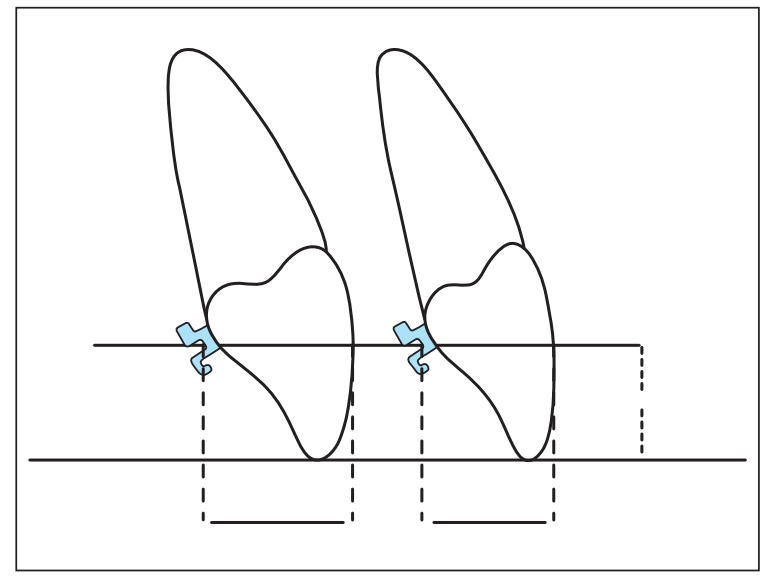

FIGURA 28 - Braquetes colados na mesma altura, porém, em dentes com diferentes espessuras. Dente à esquerda mais volumoso, no sentido vestíbulolingual comparado ao dente da direita.

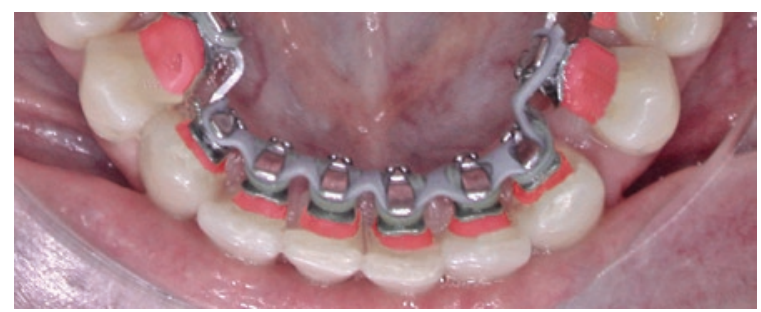

FIGURA 30 - Compensações de resina na base dos braquetes linguais inferiores.

menos efeitos colaterais em qualquer técnica ortodôntica ${ }^{11}$. Não incorrer nos erros do passado com a técnica lingual é imperioso para o sucesso com o tratamento lingual. Alem disso, é uma técnica que exige habilidade e que traz novas responsabilidades ${ }^{1,7,10,12}$.

Muito ainda precisa ser esclarecido sobre a técnica lingual e a prudência ainda continua sendo aliada do ortodontista que deseja se aprofundar no tratamento ortodôntico por lingual.

\section{CONCLUSÕES}

De acordo com a revisão literária deste trabalho, conclui-se que:

- As forças intrusivas aplicadas com a técnica lingual tendem a intruir os incisivos com menos inclinação vestibular que na técnica convencional.

- A intensidade da força produzida pelo apa-

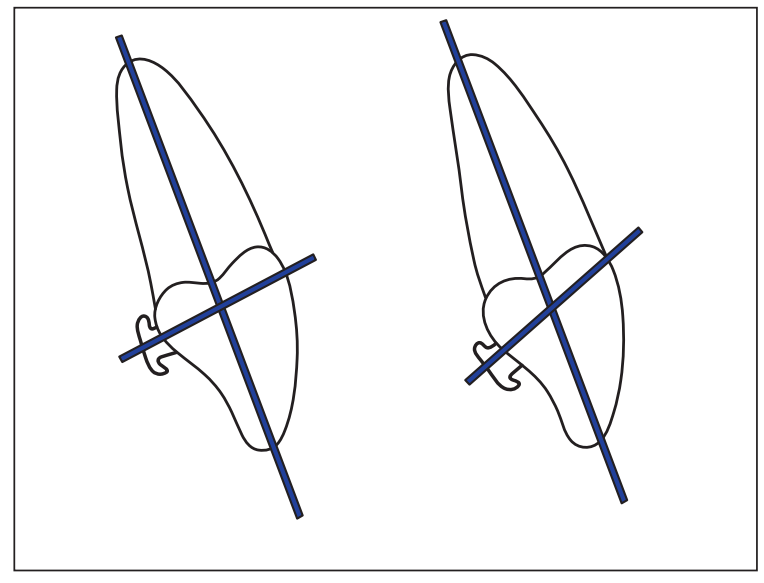

FIGURA 29 - Pequena variação de colagem do braquete afetando a inclinação do dente.

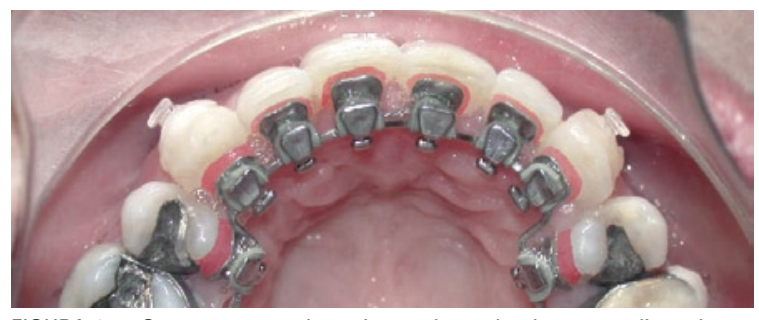

FIGURA 31 - Compensações de resina na base dos braquetes linguais superiores.

relho lingual é maior que na técnica convencional, considerando-se fios semelhantes.

- A retração pela técnica lingual tende a girar os molares mesiovestibularmente e expandir o arco na área de pré-molares.

- É praticamente indispensável a colagem indireta com compensações em resina na base dos braquetes na técnica lingual.

- A técnica lingual exige mais conhecimento sobre questões biomecânicas e habilidade do ortodontista

- Na técnica lingual há maior tendência de classe II devido ao toque anterior e abertura da oclusão posterior.

- Na técnica lingual o controle de ancoragem é mais difícil de ser conseguido.

Enviado em: fevereiro de 2006 Revisado e aceito: outubro de 2006 


\title{
Biomechanical differences between lingual and labial orthodontics
}

\begin{abstract}
Considering the lingual braces essentially esthetic they are bring up the interest of orthodontists and the general population. The increasing of the beauty services in several segments (medicine, dentistry, cosmetics etc) it is affecting the reappear of lingual technique. Due its mechanical differences comparing with buccal technique, the lingual approach demand to the orthodontist a special knowledge and training. The objective of this paper is review the literature and describes the main differences between the two techniques in order to contribute to the improvement of the orthodontists and help them to make the lingual philosophy a safe option of orthodontic treatment too.
\end{abstract}

Key words: Lingual orthodontics. Lingual appliance. Invisible brackets.

\section{REFERÊNCIAS}

1. ALEXANDER, C. M.; ALEXANDER, R. G.; GORMAN, J. C.; HILGERS, J. J.; KURZ, C.; SCHOLZ, R. P.; SMITH, J. R. Lingual orthodontics: a status report. J. Clin. Orthod., Boulder, v. 16, no. 4, p. 255-262, Apr. 1982

2. ALEXANDER, C. M.; ALEXANDER, R. G.; GORMAN, J. C. HILGERS, J. J.; KURZ, C.; SCHOLZ, R. P.; SMITH, J. R. Lingual orthodontics: A status report. Part 5. Lingual mechanotherapy. J. Clin. Orthod., Boulder, v. 17, no. 2, p. 99-115, Feb. 1983.

3. BAKER, R.W. The lingual appliance, molar eruption versus incisor depression: a cephalometric study. 1983. (Master's thesis)-Rochester, New York: Eastman Dental Center, 1983.

4. BRAUN, S.; MARCOTTE, M. R. Rationale of the segmented approach to orthodontic treatment. Am. J. Orthod.

Dentofacial Orthop., St. Louis, v. 108, no. 1, p. 1-8, July 1995

5. BURSTONE, C. J.; VAN STEENBERGEN, E.; HANLEY, K. J. Mecânica moderna do arco de canto \& a técnica do arco segmentado. São Paulo: Ed. Santos, 2003.

6. CHACONAS, S. J.; CAPUTO, A. A.; BRUNETTO, A. R. Force transmission characteristics of lingual appliances. J. Clin. Orthod., Boulder, v. 24, no. 1, p. 36-43, Jan.1990.

7. CREECKMORE, T. Lingual orthodontics-its renaissance. Am. J. Orthod. Dentofacial Orthop., St. Louis, v. 96, no. 2 p. 120137, Aug. 1989

8. DIAMOND, M. Critical aspects of lingual bracket placement. J. Clin. Orthod., Boulder, v. 17, no. 10, p. 688-691, Oct. 1983.

9. FUJITA, K. Multilingual-bracket and mushroom arch wire technique. Am. J. Orthod., St. Louis, v. 82, no. 2, p. 120-140, Aug. 1982.

10. FULMER, D. T.; KUFTINEC, M. M. Cephalometric appraisal of patients treated with fixed lingual orthodontic appliances: historic review and analysis of cases. Am. J. Orthod. Dentofacial Orthop., St. Louis, v. 95, no. 6, p. 514-520, June1989.

11. GERON, S.: ROMANO, R.: BROSH, T. Vertical forces in labial and lingual orthodontics applied on maxillary incisors - a theoretical approach. Angle Orthod., Appleton, v. 74, no. 2, p. 195-201, Apr. 2004.

12. GORMAN, J. C.; HILGERS, J. J.; SMITH, J. R. Lingual orthodontics: a status report. Part 4. Diagnosis and treatment planning. J. Clin. Orthod., Boulder, v. 17, no. 1, p. 26-35, Jan. 1983

13. GORMAN, J.; SMITH, R. J. Comparison of treatment effects with labial and lingual fixed appliances. Am. J. Orthod. Dentofacial Orthop., St. Louis, v. 99, no. 3, p. 202-209, Mar. 1991

14. HONG, R. K.; HONG, H. P.; KOH, H. S. Effect of reverse curve mushroom archwire on lower incisors in adult patients: a prospective study. Angle Orthod., Appleton, v. 71, no. 6, p. 425-432, Dec. 2001

15. KELLY, V. M. JCO Interviews on lingual Orthodontics. J. Clin. Orthod., Boulder, v. 16, no. 7, p. 461-476, July 1982.
16. KURZ, C.; BENNETT, R. Extraction cases and the lingual appliance. J. Am. Ling. Orthod. Assoc., [s.l.], v. 3, p. 10-13, 1988.

17. KURZ, C.; GORMAN, J. C. Lingual orthodontics: a status report. Part 7 - A. Case reports-nonextraction, consolidation. J. Clin. Orthod., Boulder, v. 17, no. 5, p. 310-321, May 1983.

18. KURZ, C.; SWARTZ, M. L.; ANDREIKO, C. Lingual orthodontics: a status report. Part 2.Research and development. J. Clin. Orthod., Boulder, v. 16, no. 11, p. 735-740, Nov. 1982.

19. KURZ, C. The use of lingual appliances for correction of bimaxillary protrusion (four premolars extraction). Am. J. Orthod. Dentofacial Orthop., St. Louis, v. 112, no. 4, p. 357-363, Oct. 1997

20. MORAN, K. I. Relative wire stiffness due to lingual versus labial interbracket distance. Am. J. Orthod. Dentofacial Orthop. St. Louis, v. 92, no. 1, p. 24-32, July 1987.

21. PAIGE, S. F. A lingual light-wire technique. J. Clin. Orthod., Boulder, v. 16, no. 8, p. 534-544, Aug. 1982.

22. RUMMEL, V.: WIECHMANN, D.: SACHDEVA, R. C. L. Precision finishing in lingual orthodontics. J. Clin. Orthod., Boulder, v. 33, no. 2, p. 101-113, Feb. 1999.

23. SCHOLZ, R. P. SWARTZ, M. L. Lingual orthodontics: a status report. Part 3. Indirect bonding-Laboratory and clinical procedures. J. Clin. Orthod., Boulder, v. 16, no. 12, p. 812-820, Dec. 1982

24. SMITH, J. R.; GORMAN, J. C.; KURZ, C.; DUNN, R. M. Keys to success in lingual therapy. Part 1. J. Clin. Orthod., Boulder, v. 20 , no. 4, p. 252-261, Apr. 1986.

25. SMITH, J. R.; GORMAN, J. C.; KURZ, C.; DUNN, R. M. Keys to success in lingual therapy. Part 2. J. Clin. Orthod., Boulder, v. 20 , no. 5 , p. 330-340, May 1986

26. SMITH, J. R. Lingual orthodontics: A status report. Part 7 - B. Case reports-Extraction. J. Clin. Orthod., Boulder, v. 17, no. 7, p. 464-473, July 1983.

27. SUNG, S. J.; BAIK, H. S.; MOON, Y. S.; YU, H. S.; CHO, Y. S. A comparative evaluation of different compensating curves in the lingual and labial techniques using 3D FEM. Am. J. Orthod. Dentofacial Orthop., St. Louis, v. 123, no. 4, p. 441-450, Apr. 2003.

28. TAKEMOTO, K.; SCUZZO, G. Invisible Orthodontics: current concepts and solutions in lingual Orthodontics. Germany: Quintessence Books, 2003.

29. TANNE, K.; LU, Y. C. L.; SAKUDA, M. Biomechanical responses of tooth to orthodontic forces applied at the lingual bracket positions. J. Osaka Univ. Dent. Sch., Osaka, v. 32, p. 6-13, 1992.

\section{Endereço para correspondência}

André da Costa Monini

Av. Casemiro Perez n. 560, Vila Harmonia.

CEP: 14.802-600 - Araraquara - São Paulo / SP

E-mail: amonini@hotmail.com 